\title{
Biofilm from a clinical strain of Cryptococcus neoformans activates the NLRP3 inflammasome
}

\author{
Cell Research (2013) 23:965-968. doi:10.1038/cr.2013.49; published online 9 April 2013
}

\section{Dear Editor,}

Many opportunistic fungi are devastating pathogens that cause lethal infection. Cryptococcus neoformans (C. neoformans) is such a fungus that mainly infects immunocompromised hosts such as AIDS patients as well as organ transplant recipients, and causes cryptococcosis including life-threatening meningoencephalitis [1]. Cryptococcal meningitis affects about a million AIDS patients and causes 625000 deaths in the world yearly [2]. The disease also occurs in apparently immunocompetent individuals [3]. However, host immune responses to $C$. neoformans are not well studied.

IL- $1 \beta$ is a central orchestrator in the host defense against various classes of pathogens including fungi. The secretion of IL- $1 \beta$ requires pro-IL-1 $\beta$ synthesis and cleavage by active caspase- 1 in most types of cells. The activation of caspase- 1 is largely mediated by a cellular complex called inflammasome, which contains cytoplasmic PRRs such as NLRP3, NLRC4 or AIM2, adaptor protein ASC in most cases and pro-caspase-1 [4]. As IL$1 \beta$ production via the NLRP3 inflammasome has been proved to be essential in host defense against pathogenic Candida albicans and Aspergillus fumigatus infections, and IL- $1 \beta$ production was detected from $C$. neoformansinfected mice [5-7], we hypothesized that $C$. neoformans can also activate the NLRP3 inflammasome and the latter is important for the control of $C$. neoformans infection. Indeed, in the present study, we found that biofilm from a clinical strain of $C$. neoformans induced robust IL-1 $\beta$ production from both human monocytes and murine dendritic cells in a NLRP3-dependent manner, and the NLRP3 inflammasome was essential for protection against $C$. neoformans infection.

In the current study, we applied a clinical strain of C. neoformans (HS1101) for experiments (Supplementary information, Data S1 and Figure S1). To determine whether $C$. neoformans was able to induce IL- $1 \beta$ secretion from human cells, we incubated the planktonic yeast cells with human monocytic THP-1 cells at various MOI (multiplicity of infection) for $12 \mathrm{~h}$; or at MOI $=1$ for dif- ferent time durations. However, no IL-1 $\beta$ secretion was detected (Figure 1A-1B).

As the biofilm form of fungi is usually associated with virulence [8], we therefore tested whether the biofilm form of $C$. neoformans was able to activate monocytes for IL-1 $\beta$ secretion. Surprisingly, THP-1 cell incubation with the biofilm form of $C$. neoformans resulted in a clear induction of IL-1 $\beta$ (Figure 1C). No matter through which protocol the $C$. neoformans biofilm was induced, formation of biofilm was necessary and sufficient to induce IL-1 $\beta$ secretion from THP-1 cells (Figure $1 \mathrm{C}$ and Supplementary information, Figure S2A). Moreover, the induction of IL- $1 \beta$ secretion by biofilm of $C$. neoformans from THP-1 cells was time- and dosedependent (Figure 1D-1E). Notably, induction of the IL-1 $\beta$ mRNA transcription by $C$. neoformans was also biofilm-dependent, which indicated that the biofilm of $C$. neoformans activated both NF- $\mathrm{KB}$ signaling and inflammasomes, the latter being important for maturation of IL$1 \beta$ (Supplementary information, Figure S2B). Indeed, in LPS-primed THP-1 monocytes, biofilm of $C$. neoformans induced higher levels of IL- $1 \beta$ in comparison with that in cells infected with $C$. neoformans biofilm alone (Figure 1F). Moreover, through established methods to monitor inflammasome activation [9], we observed clear ASC pyroptosome formation and caspase- 1 activation in THP-1 cells upon incubation with the biofilm form of $C$. neoformans, but not the yeast form of the same fungus (Figure $1 \mathrm{G}$ and Supplementary information, Figure S2C). Furthermore, we also found that the biofilm but not the yeast form of $C$. neoformans induced IL-1 $\beta$ production from mouse bone marrow-derived dendritic cells (BMDCs) (Figure 1H). Interestingly, production of TNF- $\alpha$ was also biofilm-dependent in these cells (Supplementary information, Figure S2D). Taken together, the biofilm but not the planktonic yeast form of $C$. neoformans induced IL-1 $\beta$ production, ASC pyroptosome formation and caspase-1 activation in myeloid cells from both humans and mice.

Next, we tested whether $C$. neoformans biofilminduced IL-1 $\beta$ secretion from THP-1 cells requires ac- 

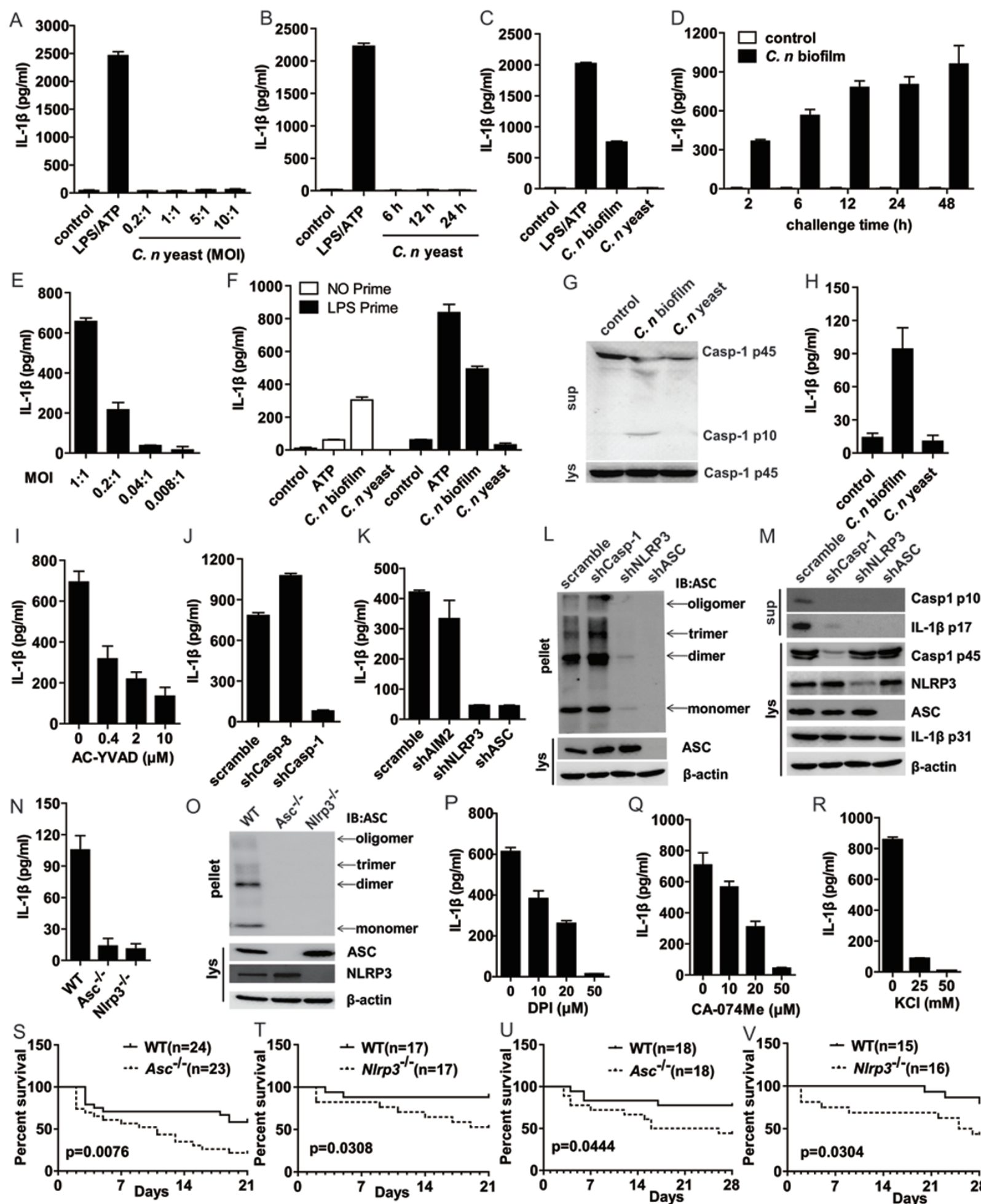

Figure 1 Cryptococcus neoformans biofilm but not the yeast form activates NLRP3 inflammasome, and the latter is essential for protecting mice from $C$. neoformans challenge in vivo. (A-B) THP-1 cell-derived macrophages were infected with the yeast form of $C$. neoformans at the indicated MOI or for the indicated time, with LPS for $6 \mathrm{~h}$ and ATP pulse for 45 min as control. The supernatants were harvested for IL-1 $\beta$ ELISA. (C) THP-1 cells were challenged with $C$. neoformans biofilm or yeast at $\mathrm{MOI}=1$, with LPS for $6 \mathrm{~h}$ and ATP pulse for $45 \mathrm{~min}$ as control. Twelve hours later, the supernatants were harvested for IL$1 \beta$ ELISA. (D-E) THP-1 cells were treated with $C$. neoformans biofilm at $\mathrm{MOI}=1$ for the indicated time or at the indicated $\mathrm{MOI}$ for $12 \mathrm{~h}$; IL-1 $\beta$ in supernatants was detected via ELISA. (F) THP-1 cells were primed with LPS (10 $\mathrm{ng} / \mathrm{ml})$ for $4 \mathrm{~h}$ and then 
tivation of caspase- 1 given that caspase- 1 activation is needed for the maturation of IL-1 $\beta$ in monocytes and macrophages. To this end, a caspase-1-specific inhibitor AC-YVAD was applied in the $C$. neoformans biofilm stimulation experiments. We found that AC-YVAD abolished IL-1 $\beta$ secretion in a dose-dependent manner (Figure 1I) without affecting inflammasome-independent IL-8 production (Supplementary information, Figure S3A), indicating that caspase-1 activity was necessary for $C$. neoformans biofilm-induced IL- $1 \beta$ release. This was further confirmed via shRNA-mediated silencing of caspase-1 in THP-1 cells, wherein silencing of caspase-1 also completely abolished $C$. neoformans biofilminduced IL-1 $\beta$ secretion (Figure $1 \mathrm{~J}$ ). Of interest was that caspase-8, which was involved in the responses to various fungal challenges [10], seemed to be not required for C. neoformans biofilm-induced IL-1 $\beta$ secretion, indicating that specific signaling pathway accounted for the IL$1 \beta$ induction by different fungi. In addition, knockdown of caspase- 8 showed elevated secretion of IL-1 $\beta$ upon $C$. neoformans biofilm challenge, which demonstrated for a regulatory role of caspase- 8 in inflammasome activation under certain circumstances as revealed from a recent study [11]. As caspase-1 activation is largely dependent on assembly of inflammasomes, we sought to determine which inflammasome was specifically involved in the response to C. neoformans biofilm stimulation. As shown in Figure 1K, silencing of AIM2 did not change the induction of IL-1 $\beta$ by $C$. neoformans biofilm. However, in sharp contrast, silencing of ASC or NLRP3 resulted in the complete loss of IL- $1 \beta$ secretion (Figure $1 \mathrm{~K}$ ). In addition, ASC pyroptosome formation and caspase- 1 activation induced by the $C$. neoformans biofilm was also NLRP3 inflammasome-dependent (Figure 1L and 1M). Of note was that silencing of caspase- 1 did not affect the formation of ASC pyroptosome, implying that the latter is a pre-step for caspase-1 activation instead of coincidence, which was also indicated by similar experiments performed in caspase-1-deficient mouse macrophages in our recent study [9]. The efficiency of shRNA-mediated gene silencing was well controlled, and IL-8 secretion was largely unaffected by these silencing (Figure $1 \mathrm{M}$ and Supplementary information, Figure S3B-S3D). Moreover, inflammasome activation induced by $C$. neoformans biofilm in mouse BMDCs was also NLRP3dependent, as BMDCs from Nlrp3-or Asc-deficient mice were completely defective for IL- $1 \beta$ secretion and ASC pyroptosome formation upon such infection, while TNF- $\alpha$ secretion was not affected (Figure $1 \mathrm{~N}-1 \mathrm{O}$ and Supplementary information, Figure S3E). Thus, induction of IL-1 $\beta$ by $C$. neoformans biofilm in either THP1 cells or BMDCs was dependent on the activity of both caspase-1 and the NLRP3 inflammasome.

A feature of the NLRP3 inflammasome activation is that multiple upstream stimuli lead to ROS production and potassium $\left(\mathrm{K}^{+}\right)$efflux, and these common downstream factors result in the activation of caspase- 1 . To test the role for each of these factors in C. neoformans biofilm-induced NLRP3 inflammasome activation, we applied ROS scavenger DPI in THP-1 cell cultures incubated with $C$. neoformans biofilm. It was found that DPI inhibited IL-1 $\beta$ production in a dose-dependent manner (Figure 1P). Of note was that DPI treatment affected the production of IL-8 similarly, which was in line with a recent study showing that ROS inhibitors, including DPI, interfere with NF- $\kappa \mathrm{B}$ signaling (Supplementary information, Figure S4A) [12]. Moreover, as Cathepsin $B$ activity was also involved in the response of various stimulus-induced inflammasome activation [13], we tested the possibility that it might be also required for $C$. neoformans biofilm-induced NLRP3 inflammasome activation. Indeed, Cathepsin B inhibitor CA-074 Me clearly interfered with the production of IL- $1 \beta$ from THP-1 cells upon $C$. neoformans biofilm infection in a dose-dependent manner (Figure 1Q), while Cathepsin $\mathrm{K}$ inhibition showed little effect (Supplementary information, Figure

co-cultured with $C$. neoformans biofilm or yeast for $6 \mathrm{~h}$; IL-1 $\beta$ in supernatants was then detected via ELISA. (G) THP-1 cells were challenged with $C$. neoformans biofilm, or yeast at $\mathrm{MOI}=1$. Twelve hours later, caspase- 1 activation was detected with immunoblotting. (H) BMDCs from WT mice were challenged with $C$. neoformans biofilm, yeast or medium control at MOI = 1 for $12 \mathrm{~h}$ and the supernatants were harvested for IL-1 $\beta$ ELISA. (I) THP-1 cells were pretreated with AC-YVAD and then challenged with $C$. neoformans biofilm for $12 \mathrm{~h}$ and the supernatants were harvested for IL-1 $\beta$ ELISA. (J-K) THP-1 cells with shRNA silencing of the indicated genes were treated with $C$. neoformans biofilm and IL-1 $\beta$ was detected via ELISA. (L-M) Mature form of caspase-1 ( $\mathrm{p} 10$ ) and mature IL-1 $\beta$ ( $\mathrm{p} 17$ ) in culture supernatants (sup) and other indicated proteins in cell lysates (lys) as well as ASC pyroptosome formation from the indicated cells stimulated with $C$. neoformans biofilm, were analysed via immunoblotting. (N-O) BMDCs from WT, NLRP3- or ASC-deficient mice were challenged with $C$. neoformans biofilm at MOI = 1 for $12 \mathrm{~h}$, and then the supernatants were harvested for IL-1 $\beta$ ELISA and ASC pyroptosome formation was detected through immunoblotting. (P-R) THP-1 cells were pretreated with DPI, CA-074 Me or KCl, then challenged with C. neoformans biofilm, and the supernatants were harvested for IL-1 $\beta$ ELISA. (S-V) The indicated mice were challenged with i.p. injection (S-T) or i.n. infection of C. neoformans (U-V) and monitored daily for survival. C. neoformans is abbreviated as $C$. $n$ in the figures. 
S4B) [14]. All these above treatments did not reduce but slightly elevated the production of IL-8 (Supplementary information, Figure S4C-S4D). As expected, potassium efflux inhibition also abolished C. neoformans biofilm induced IL-1 $\beta$ secretion (Figure 1R). Although IL-8 level was decreased when the cells were treated with higher concentration of $\mathrm{KCl}$, the extent of decrease was much less than that for IL-1 $\beta$ (Supplementary information, Figure S4E). The moderate effect of $\mathrm{KCl}$ on IL-8 secretion could result from osmotic stress in THP-1 cells.

All these above in vitro experiments showed that $C$. neoformans biofilm activated the NLRP3 inflammasome. To test the importance of NLRP3 inflammasome in combating $C$. neoformans infection in vivo, we infected mice deficient for Nlrp3 or Asc through intraperitoneal (i.p.) and intranasal (i.n.) routes. It was found that in the absence of either of these molecules, the death rate of mice was significantly higher than in wild type (WT) control (Figure 1S-1V). Moreover, upon C. neoformans infection, the death of $A s c$ - and Nlrp3-deficient mice took place much earlier. This indicated that in the early phase of infection, activation of the NLRP3 inflammasome is very important to confine the colonization and spreading of $C$. neoformans. Although part of the WT mice eventually died from this infection, the event was much delayed (Figure 1S-1V). These in vivo experiments thus established an essential role for the NLRP3 inflammasome in host defense against $C$. neoformans infection.

Our work thus discovered that the NLRP3 inflammasome was able to sense and respond to $C$. neoformans biofilm; moreover, our work uncovered that inflammasome is an important arm for host defense against $C$. neoformans infection, and suggests that manipulating NLRP3 signaling may help to oppose $C$. neoformans infection in patients.

\section{Acknowledgments}

We thank Dr Warren Strober (National Institutes of Health, USA) for providing the Nlrp3-deficient mice, Dr Vishva Dixit (Genentech, Inc., USA) for providing Asc-deficient mice, Dr Jürg Tschopp (University of Lausanne, Switzerland) for providing shRNA constructs. We also thank Dr Xudong Zhu (Nankai University, China) for helpful discussion and our colleague Ms Cuili Dong for excellent technical assistance. This work was supported by grants from 100 Talent Program of the Chinese Acad- emy of Sciences, the National Natural Science Foundation of China (91029707, 31170868, 812111134, 31100622), Shanghai Natural Science Foundation (11ZR1442600), Novo Nordisk-CAS Research Foundation, SA-SIBS Scholarship Program, Chinese Postdoctoral Science Foundation (20110490752) and Postdoctoral Research Foundation of Shanghai Institutes for Biological Sciences (2011KIP513), as well as grants from the National Key Basic Research Program (2013CB531600) and National Science and Technology Major Project (2012ZX10002007-003) of China.

Guowei Lei ${ }^{1,2, *}$, Mingkuan Chen ${ }^{1, *}, \mathrm{Hua} \mathrm{Li}^{1,2, *}$, Jun-Ling $\mathrm{Niu}^{3}$, Shuxian $\mathrm{Wu}^{1}$, Liming $\mathrm{Mao}^{1}$, Ailing Lu ${ }^{1}$, Hongbin Wang ${ }^{1}$, Wei Chen ${ }^{1}$, Bin $\mathrm{Xu}^{4}$, Qibin Leng ${ }^{1}$, Cunshuan $\mathrm{Xu}^{3}$, Guiwen Yang ${ }^{2}$, Liguo $\mathrm{An}^{2}$, Li-Ping Zhu ${ }^{4}$, Guangxun Meng ${ }^{1}$

${ }^{I}$ Key Laboratory of Molecular Virology and Immunology, Institut Pasteur of Shanghai, Shanghai Institutes for Biological Sciences, Graduate University of Chinese Academy of Sciences, Shanghai 200025, China; ${ }^{2}$ College of Life Science, Shandong Normal University, Jinan, Shandong 250014, China; ${ }^{3}$ College of Life Science, Henan Normal University, Xinxiang, Henan 453007, China; ${ }^{4}$ Department of Infectious Diseases, Huashan Hospital, Fudan University, Shanghai 200040, China

*These three authors contributed equally to this work. Correspondence: Guangxun Meng ${ }^{\mathrm{a}}$, Li-ping $\mathrm{Zhu}^{\mathrm{b}}$

${ }^{\mathrm{a}}$ Tel/Fax: +86 2154652203

E-mail: gxmeng@sibs.ac.cn

${ }^{\mathrm{b}}$ Tel/Fax: +86 2152887966

E-mail: zhulp@fudan.edu.cn

\section{References}

1 Del Poeta M, Casadevall A. Mycopathologia 2012; 173:303-310.

2 Park BJ, Wannemuehler KA, Marston BJ, et al. AIDS 2009; 23:525530.

3 Zhu LP, Wu JQ, Xu B, et al. Med Mycol 2010; 48:570-579.

4 Franchi L, Munoz-Planillo R, Nunez G. Nat Immunol 2012; 13:325332 .

5 Hise AG, Tomalka J, Ganesan S, et al. Cell Host Microbe 2009; 5:487497.

6 Said-Sadier N, Padilla E, Langsley G, et al. PLoS One 2010; 5:e10008.

7 Barluzzi R, Brozzetti A, Mariucci G, et al. J Neuroimmunol 2000; 109:75-86.

8 Fanning S, Mitchell AP. PLoS Pathog 2012; 8:e1002585.

9 Mao K, Chen S, Chen M, et al. Cell Res 2013; 23:201-212.

10 Gringhuis SI, Kaptein TM, Wevers BA, et al. Nat Immunol 2012; 13:246-254.

11 Kang TB, Yang SH, Toth B, et al. Immunity 2013; 38:27-40.

12 Bauernfeind F, Bartok E, Rieger A, et al. J Immunol 2011; 187:613617

13 Schroder K, Tschopp J. Cell 2010; 140:821-832.

14 Allen IC, Scull MA, Moore CB, et al. Immunity 2009; 30:556-565.

(Supplementary information is linked to the online version of the paper on the Cell Research website.) 